\title{
Seamless Communication Network for Enhanced Performance in Tunnel Based High Speed Trains using Q-Leach and Fodpso Algorithm
}

\author{
S. Priyanka, S. Leones Sherwin Vimalraj, J.Lydia
}

\begin{abstract}
Wireless Communication on Train (WCT) is being followed in urban railways around the world to enhance the railway network efficiency, safety, and capacity which are mainly carried in high speed trains and underground tunnels. It is mainly used in underground tunnels where there is a large amount of reflections, scattering and barriers are severely affecting the propagation performance. The researchers have introduced Low Energy Adaptive Clustering Hierarchy (LEACH) protocols for efficient data gathering with efficient routing to reduce the energy consumption in the wireless network. We propose Quadrature-LEACH $(Q-L E A C H)$ protocol for heterogeneous network to improve the routing efficiency and throughput significantly with optimal functionality of network lifetime. The heterogeneous network consist of Wi-Max, Wi-Fi and LTE for real time application, the network should be highly equipped with a robust, reliable and efficient routing protocol so that the packets could be routed on the network with lower latency. The FODPSO (Fractional Order Darvinian Particle Swarm Optimization) optimization algorithm is applied to improve the performance of heterogeneous network for data handover and efficient routing. The optimization algorithm is used for the reliability of wireless link quality and the bandwidth utilization on the wireless routing path. The experimental results are proven that the optimal routing and higher range of link quality between various networks with low latency to minimize the time delay of data handover.
\end{abstract}

Keywords: High Speed Train, Tunnel, Q-Leach, FODPSO, Heterogeneous networks

\section{INTRODUCTION}

Heterogeneous access network has increased opportunities with technological progresses in wireless communication system and mobile device by the multipath data transfer. Real time video streaming over HWN utilized bandwidth - efficient multipath streaming for the process.

Revised Manuscript Received on November 22, 2019.

S. Priyanka, Department of Electronics and Communication Engineering, Panimalar Engineering College, Chennai, India, Email:saravananpriyanka95@gmail.com

Dr Leones Sherwin Vimalraj, Department of Electronics and Communication Engineering, Panimalar Engineering College, Chennai, India. Email: leonessherwin@gmail.com

J.Lydia , Department of Electronics and Communication Engineering, Easwari Engineering College, Chennai, India. Email:lydia_822@yahoo.co.in
Bandwidth-efficient multipath streaming is primarily used to decrease technical problems linked to standard multipath protocols video information is programmed in a bandwidth-intensive content-agnostic style and high quality real time and susceptible to delay and has forward error correction based accurate transmission [1]. Framework is used to perform an effective traffic flow in heterogeneous network. By splitting the traffic flow, the best network is selected which has the minimized delay. The data packet performance is analyzed by Markov chain [2]. E-STAR (Establishing Stable and reliable Routes) joines the payment and trust system for efficient routing. The nodes are charged by the credits and trust party (TP) manage their accounts. The node submits the receipts to the TP. Trust system is performs the key authentication, electronic commerce and supporting decision making. The past behaviour of node is used to calculate the trust values and it used to predict their future behaviour [3]. The standard technology of WiMAX (World interoperability for Microwave Access) and WiFi are of different frequency. The CPU clock is used for WiFi and WiMAX communicate with each other. WiFi has multi-vendors. For WiMAX and WiFi convergence the difference between two technologies have been evaluated WirelessMan-SCa PHY specification is considered for WiMAX and WiFi convergence (mixed-standard) [4]. Heterogeneous network (HetNet) consist of WiFi and WiMAX which are used in Internet of Things (IOT). WiMAX is used to improve QoS and WiFi is used in reduced power consumption. Arbitration inter frame space number (AIFSN) for channel gain access [5].Lyapunov optimization technique is used to develop quality-aware streaming algorithm to maximize the network utility. The determination of power allocation and packet scheduling are developing the content-aware framework and minimize the quality distortion. The quality aware streaming for heterogeneous network with time varying channels adjust the quality for HWN [6]. Adaptive technology is used to improve the performance of wireless device in terms of high throughput and low delay. The throughput optimal control strategies are minimizing the average power expenditure. The cell portioned is simplifies the exposition and perform decoupled cell by cell using 
scheduling decisions. Power allocation is categorized into dynamic control algorithm it develop the energy efficient control strategies with performance[7]. The data gathering in wireless networks have a technical issue of energy hole problem to it performance. Sensor with static sink act as relays depletes the energy as fast in the sensor field that shows the energy hole in the sensor. Mobile Sink based adaptive Immune Energy-Efficient clustering Protocol (MSIEEP) is used the adaptive immune algorithm (AIA) to reduce the energy holes in the sensor field. The algorithm is mainly required for guide the mobile sink and to determine the optimum number of cluster head (CHs). It improves the stability period and life time of network. The sensor measurements such as heat, pressure, vibration, presence of objects are forwarded towards a static sink. Premature disconnection of the network is occurs due to the energy hole problem. Clustering algorithm is used for avoid the energy hole problem. Adaptive immune algorithm is to find the sojourn locations of mobile sink. The MSIEEP is more reliable and energy efficient then other protocols [8]. Thousands of sensor nodes work together to construct the high quality fault tolerant sensor network. Redundant data from multiple nodes of wireless network are combined to reduce transmission. Energy consumption is done from transmitting or receiving data messages, and processing requests or from the operation of constructing routing tree, overhearing, retransmitting. GSTEB is providing simple approach to balancing the network load. GSTEB is compute topography by using the base station [9]. The Quality of service is mainly along with mobility of aware admission control scheme are used energy efficient offloading and integrates a novel bandwidth. Time varying call traffic is used to find the optimal solution of the problem. Free unlicensed spectrum is needed for WiFi and LTE for good performance and minimization of energy consumption. [10].

\section{BLOCK DIAGRAM}

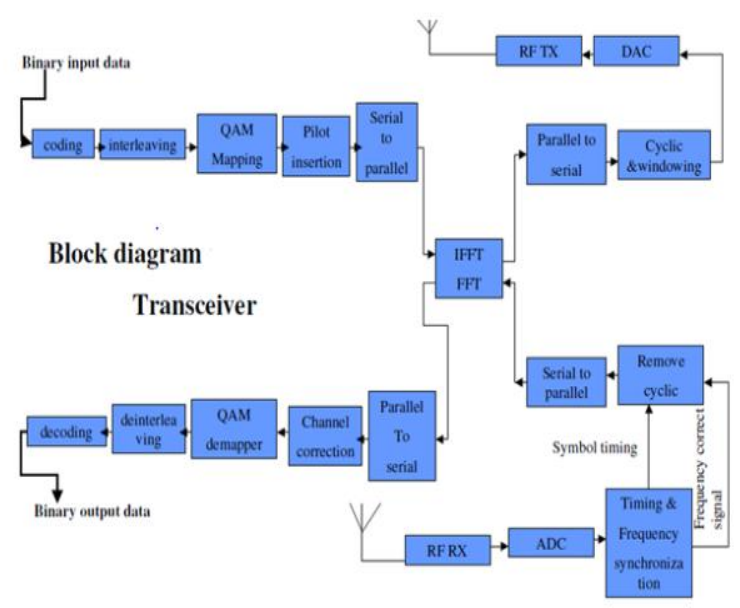

Fig. 1. Block Diagram of WCT

In Figure 1, To generate simple random data originally and then use the orthogonality conversion function. In this function, the data can be converted into one format and minus one. Linear dimensions of $\mathrm{M}$ pour $\mathrm{N}$ are intended to be the sparse signal. Time delay happens when the signal is sparse. This time restriction is thus avoided by microsecond latency. The sparse signal is converted into a continuously complex exponential sinusoidal signal. This signal is used for transportation on long distances. Apply twiddle factor to that post. A twiddle factor is a fast Fourier algorithm for signal transformation and twiddle factor estimation. Apply the channel MIMO. There are two streams in this MIMO channel, AWGN and Rayleigh. These two channels pass the scarce signal. After that, the spatial and temporal correlation of the structure must be calculated. The equalizer and heuristic approach used to calculate the mean square error, SNR and capacity

\section{RELATED WORKS}

Rajarajan Sivaraj, Aravind Kota Gopalakrishnay et.al [11] have presented Cluster Head (CH) to manage vehicular Ad hoc networks (VANET) for universal mobile telecommunication system (UMTS). Long term evolution (LTE) gives uplink and downlink data rates. Eleni Stai 1 and Michail Loulakis2 et.al [12] have presented the NUM framework multihop networks. Stochastic Differential Equations (SDE) is used to create a wireless channel power loss. The design and optimization network are designed by Network Utility Maximization tool. When the power control is applied to reduce the loss then it considered the energy efficiency. SIR (signal- to-interference radio) is expressing the channel quality. The interference parallel transmission and LTE is can affect the scatter. LTE is use the SDEs for express the LTE channel model capturing both space and time variation. Power control for higher source rate is improves the energy efficiency. Duc Chinh Hoang and Parikshit Yadav et.al [13] have presented the framework for centralized cluster-based protocols for WSNs. The process of optimization is improves the life time of the network. Meta-heuristic optimization method is designed and implemented for WSN and it optimized the energy distribution of the WSN. The sensors from the WSN have low power, small size and it works as autonomous device. The renewable energy source is integrated with sensor nodes for the life time development of WSN. The operation of WSNs based cluster routing protocols have higher energy efficient. The group of sensor nodes called the cluster each node from the cluster called cluster hear $(\mathrm{CH})$ it transfer the data information to Base Station. LEACH is a typical cluster based protocol is uses a centralized clustering mechanism to form the cluster. Yan Jiao and Inwhee Joe et.al [14] have presented the algorithm to get optimal solution in power and bandwidth. The radio environment map is managed the resource allocation and network synchronization. It is stable and fast convergence. Cognitive radio (CR) is required for scarcity problems in spectrum. Capacity is increased by muti radio access technology. The femtocell technology is provides improved cellular coverage to the user equipment. The EERA method is maximize the system downlink capacity over lapping areas. The EERA is an optimal resource allocation solution. Joohyung Lee and Nga T. Dinh et.al [15] have presented the efficient load distribution in multipath. It is used power 
consumption and delay of interference. The load balancing from heterogeneous wireless access network is done by using Multihoming. The multi interfaced handheld devices provides multimedia content anytime anywhere to the users. The QOs is addressed from the approach of realizing efficient load distribution solution that approach have drawback such as load imbalance and packet reordering. The multihomed MTs is minimize the power consumption using the framework. The analytical model is used for various interfaces in the MT. Illsoo Sohn and Jong-Ho Lee et.al [16] have presented the low energy adaptive clustering hierarchy $(\mathrm{LEACH})$ protocol for wireless network. It utilized the cluster formation based on affinity propagation (PG). The recharging of the battery in wireless sensor network is impossible without the sophisticated coordination therefore it need energy management for maximizing their life time. The multiple cluster of the node formed by the low-energy adaptive clustering hierarchy (LEACH) Protocol which designate a single cluster head $(\mathrm{CH})$ node in each cluster for minimize the energy consumption of WSNs. To determine the temporal variation in network topology is difficult with optimal cluster number. Amina Gharsallah and Nouri Omheni et.al [17] have presented decision making during handover. The integration of wireless network is requires the improvement. Vertical handoff is an effective issue of the heterogeneous network. The 802.21 standard (MIH) make a link layer intelligence for facilitate the integration of heterogeneous network. It helps the handover decision. Yongjun Sun and Wenxin Dong et.al [18] have presented the routing using ant colony algorithm. It is used to determine the optimal path from source node to the destination node that it reduces the network energy consumption and prolong the network lifetime. WSN nodes have limited energy due to powered by battery. The algorithm is used for optimization process of the WSN, it minimize the energy consumption. The algorithm complexity is increased by the penalty function. To apply ant colony routing algorithm is leads to high average and a minimal residual energy level. It extends the life time of the wireless network. Vlad Tabus and Dmitri Moltchanov et.al [19] have presented the suboptimal design. The chain topology in chain routing with even energy consumption (CREEC) is used. The communication of network occurs between the same pairs of nodes it keeps topology reconfiguration as minimum. The optimal scheduling is used to maximize the network lifetime. Combination of chains with upper bounded is obtains the maximum lifetime. The energy consumption is depends on the two important factors such as performance of motes and distance. The two-stage star topology is transmitting the information in the wireless sensor network. The sophisticated hierarchical structure is used to hierarchical power efficient gathering in sensor information system. The power efficient data gathering is done through the non-hierarchical tree topology. Spanning tree algorithm is minimizing the energy consumption. The upper bound on the achievable lifetime is used to understand the range of possible solution. Rejina Parvin and Vasanthanayaki C et.al [20] have presented the PSO. The E-OEERP is terminates the some formation of nodes and increases the life time of the network by the concept of particle swarm optimization (PSO) with Gravitational search algorithm. The supportive node of each cluster head is called as cluster assistant (CA). Single-hop transmission, Multi-Hop transmission, Cluster based transmission, Tree based and Chain based transmission. The next best hop is determined by using the gravitational search algorithm.

\section{THE PROPOSED ROUTING PROTOCOL OPTIMIZATION IN HETEROGENEOUS WIRELESS NETWORKS}

The Q-LEACH energy efficient routing protocol for heterogeneous wireless networks, a system of routing the data is proposed with FODPSO optimization. The energy dissipation during the transmission from cluster head to the base station can be minimized to improve the routing efficiency. The heterogeneous wireless network (HWN) consists of three networks named Wi-Max, Wi-Fi and LTE system for data communication. The new Q-LEACH routing protocol achieves above mentioned requirement to improve the network efficiency with various parameters.

\section{Q-LEACH ROUTING PROTOCOL}

The proposed system named as Q-LEACH. This protocol is the key concept of proposed system. By using clustering, stability and network life-time for optimization. Clustering is done by dividing the network area for better coverage. Moreover, it conjointly presents an idea of efficient clustering mechanism that yields considerably in higher coverage of entire network. The deployed random nodes in a very $100 \mathrm{~m} \times 100 \mathrm{~m}$ filed. Supported location data, is divided into four equal quadrants such as a1,a2,a3,a4.

$\mathrm{A}=\mathrm{a} 1+\mathrm{a} 2+\mathrm{a} 3+\mathrm{a} 4$

an $=\mathrm{A}(\mathrm{xm}, \mathrm{ym})$

Let us consider

$\mathrm{n}=4$, it is no of users

$\mathrm{m}=100$, th coverage distance

Where overall field distribution

$\mathrm{Ym}=0: 50 \operatorname{limXm}=0: 50 \mathrm{an}+\mathrm{Ym}=0: 50 \operatorname{limXm}=51: 100 \mathrm{an}+\mathrm{Ym}$ $=51: 100 \operatorname{limXm}=0: 50 \mathrm{an}+\mathrm{Ym}=51: 100 \operatorname{limXm}=51: 100 \mathrm{an}$ (2)

\section{A. Algorithm 1 Initial Phase}

The Figure 2 describes the method for selecting CHs. The overall network is divided into four fields: A, B, C and D room. Each node initially chooses whether or not to become a $\mathrm{CH}$. Node selects a random variety from zero to zero. If this range is less than the bound threshold $\mathrm{T}(\mathrm{n})$, and the condition for the desired range of $\mathrm{CHs}$ is not met during a specific space, then the node becomes a $\mathrm{CH}$. For the rest of the sectors, the same method continues and the optimum range of clusters is shaped. Cluster selection may be based on the advertisement's Received Signal Strength Indicator (RSSI). Once clusters are called, nodes should notify $\mathrm{CHs}$ of their connection. On the premise of gathered data from attached up 
nodes, desired time slots are allocated to nodes using TDMA

1: begin
2: if node $\varepsilon \mathrm{G}-\rightarrow \mathrm{G}=$ nodes which did not become
CHs in current EPOCH then
3: if (NODE BELONGS TO ==_areaA) then
4: if (NUMBEROFCHs \&lt; $=$ NK_) then
5: TEMP=random number (0-1)
6: if (temp \&lt; $=$ P1-P(r,mod $1 / \mathrm{P})$ ) then
7: node=CH A
8: NUMBER OF CHs $=$ NUMBER OF CHs +1
9: end if
10: else if (NODE BELONGS TO ==areaB) then
11: REPEAT STEP $4: 8$
12: else if (NODE BELONGS TO == areaC) then
13: REPEAT STEP $4: 8$
14: else if (NODE BELONGS TO ==areaD) then
15: REPEAT STEP $4: 8$
16: end if
17: end if

Fig. 2. Initial Phase

\section{B. Algorithm 2 Node Connection with relevant $\mathrm{CHs}$}

Figure 3 identifies node connection with relevant CHs. Non- $\mathrm{CH}$ nodes can be found in the room to which they belong. Then they will search for all achievable $\mathrm{CHs}$ and start affiliation on RSSI's assumption. This technique can proceed until portion of the organization includes finishing of the organization. Once the cluster configuration portion is finished and nodes are allocated to each node with TDMA slots at their allocated quantity. Any non-cluster head node's time radio can remain off to optimize energy usage. Once the $\mathrm{CHs}$ receive all node data, the data is condensed and sent to BS. For the next spherical, the spherical complete and new choice of $\mathrm{CHs}$ are initiated. In the scheduled scheme, we have a tendency to use the same radio model as stated for the transmission and receipt of information from sensor nodes to $\mathrm{CHs}$ in addition to the stated idea of localized cooperation in each sector area.

An efficient-energy Q-LEACH is used for data gathering for routing protocol. It is used for energy consumption and increases network longevity. The flow of EE-LEACH Protocol is shown in Figure 4.

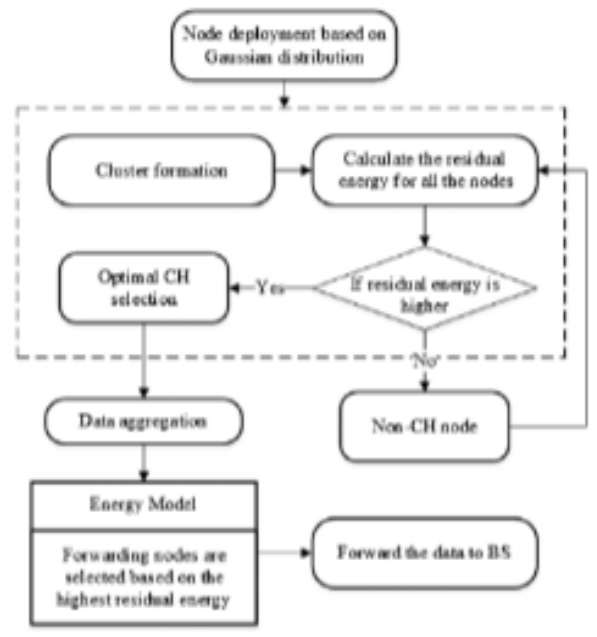

Fig.4. Energy Efficient Q-LEACH Protocol
1: $\mathrm{N} \in$ Group of normal nodes
2: $\mathrm{GC} \in$ Group of $\mathrm{CHs}$
3: if $\mathrm{N} \in(\mathrm{A}, \mathrm{a} 1)$ then
4: Where
5: $\mathrm{A}=\mathrm{a} 1, \mathrm{a} 2, \mathrm{a} 3, \mathrm{a} 4$
6: Check all possible ACHs
7: Check RSSI of CHs
8: Associate with ACHs
9: then
10: transfer of data occurs
11: end if
12: if $\mathrm{N} \in(\mathrm{A}, \mathrm{a} 2)$ then
13: Repeat step from $5: 8$ for $\mathrm{BCHs}$
14: end if
15: if $\mathrm{N} \in(\mathrm{A}, \mathrm{a} 3)$ then
16: Repeat step from $5: 8$ for $\mathrm{CCHs}$
17: end if
18: if $\mathrm{N} \in(\mathrm{A}, \mathrm{a} 4)$ then
19: Repeat step from $5: 8$ for DCHs
20: end if

Fig. 3. Node Connection with relevant $\mathrm{CHs}$

The Figure 5 shows the heterogeneous network clustering model for different networks clustering. In out proposed system, Wi-Max, Wi-Fi and LTE networks are connected in heterogeneous model. The capability of wireless communication systems may be more improved through innovation and by optimizing the network schemes and infrastructures to accommodate the subsequent generation multimedia system wireless traffic needs. Seamless communication may be achieved by integration short rang wireless networks and wide rang wireless networks such as Wi-Max, Wi-Fi and LTE.

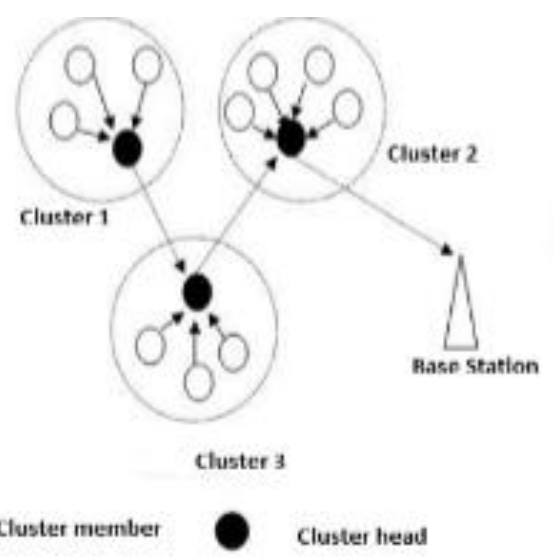

Fig..5. Heterogenous Network Clustering Model

\section{PSO OPTIMIZATION FOR ENERGY ROUTING}

The FODPSO is the modified optimization algorithm of PSO. In our proposed system, FODPSO is used to optimize the performance of routing system of Q-LEACH protocol for 
heterogeneous wireless network. In FODPSO system, where every particle searches and adjusts its position in search area consistent with its own expertise which of neighbouring particles. The options of the method are as follows:

1) It searches nodes by bird flocking

2) Easy conception is supported and computation time is brief

In bird flocking, birds find food by flocking not by each individual. Where it relates the behaviour of human groups and behaviour pattern of every individual that experiences by every individual. These are the basic ideas of FODPSO. Every agent is aware of its best worth so far (pbest) and its sex chromosome position. Each agent knows the simplest worth to date within the cluster ( gbest) among pbest

The distance between this position, and pbest and gbest are estimated with velocity:

$\operatorname{Vi}(\mathrm{t}+1)=\left[\mathrm{w}^{*} \mathrm{vi}(\mathrm{t})+\mathrm{C} 1 * \operatorname{rand}() *(\right.$ pibest $-\mathrm{xi}(\mathrm{t}))+\mathrm{c} 2 * \operatorname{rand}() *(\mathrm{gbe}$ st-xi(t))]

Using the higher than equation, a particular rate that bit by bit gets near pbest and gbest will be calculated by:

$\mathrm{Xi}(\mathrm{t}+1)=\mathrm{xi}(\mathrm{t})+\mathrm{vi}(\mathrm{t}+1)$

\section{A. The FODPSO Algorithm:}

Input: Randomly initialized position and velocity of the particles:

Output: Position of the approximate global optimal optima

Begin

While terminating condition is not reached do

Begin

For i 1 to number of particle

Evaluate the fitness value

Update pbest and gbest

Adapt velocity of the particle using eq. (3);

Update the position of the particle using eq. (4);

Increase i;

End For

End While

Fig. 6. The FODPSO Algorithm

In Figure 7, The probability of detection is said to be efficient, only if it is 1 . The power factor or false alarm is been given with three factors $0.05,0.03$ and 0.01.As the SNR increases probability of detection also increases. In Figure 8, The Bit Error Rate is said to be efficient, only if it is 0.1 . The Heterogenous Network is been given with three factors Wimax, LTE And Wifi. Latency Minimization using FODPSO Optimization is said, as the SNR increases the bit error rate also increases. In Figure 9, The Link Quality is said to be efficient only it is 1 . For Network Distance Of 100 meters the link quality is said to be 1 as the link quality increases network distance also increases. In Figure 10, The Packet Loss found in the heterogenous networks where the capacity is found to be decreased as the threshold point is reached. For packet loss vs capacity, the packet loss increases as there is a increase in capacity.

\section{RESULT AND DISCUSSION}

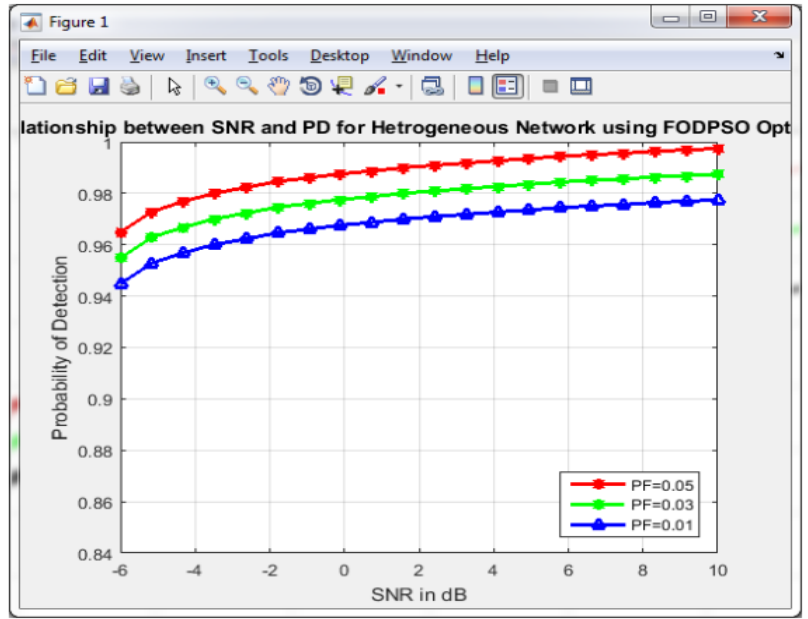

Fig.7. Relationship between SNR and PD for Heterogenous Network

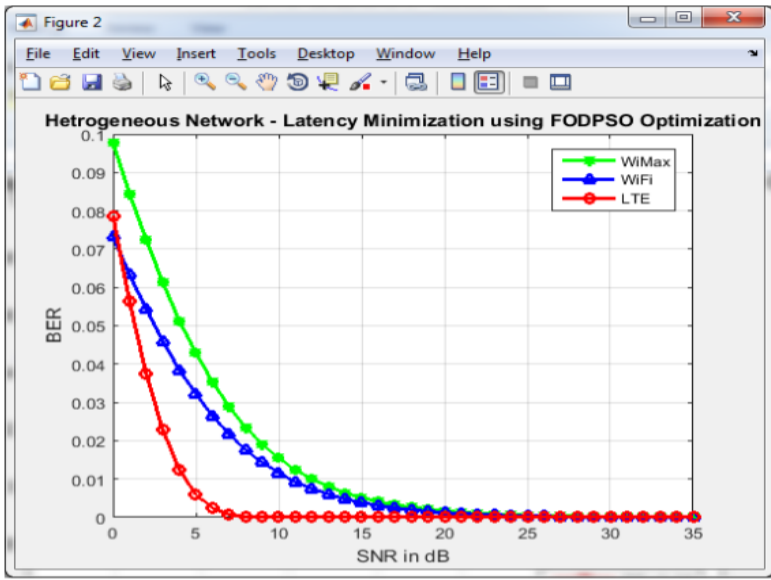

Fig.8. Heterogenous Network- Latency Minimization

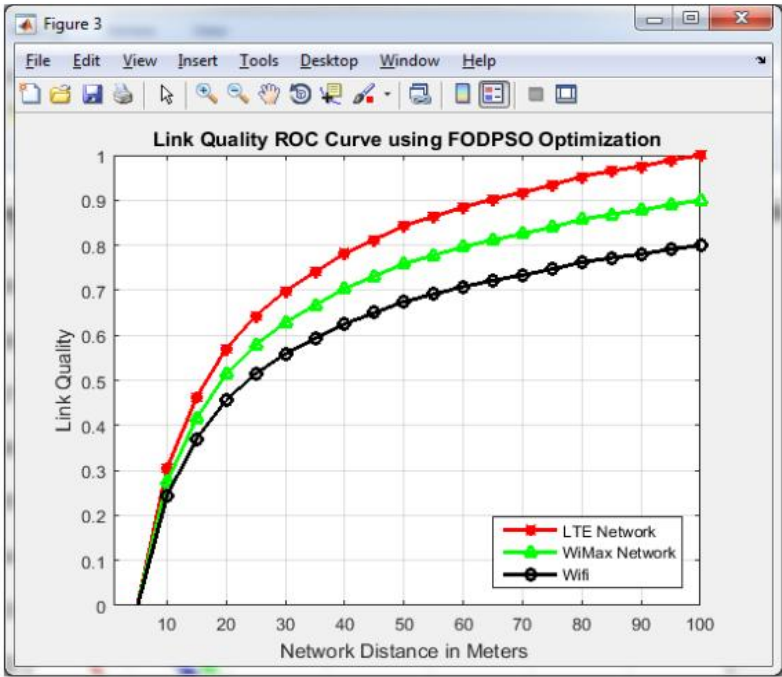

Fig.9. Link Quality ROC Curve 


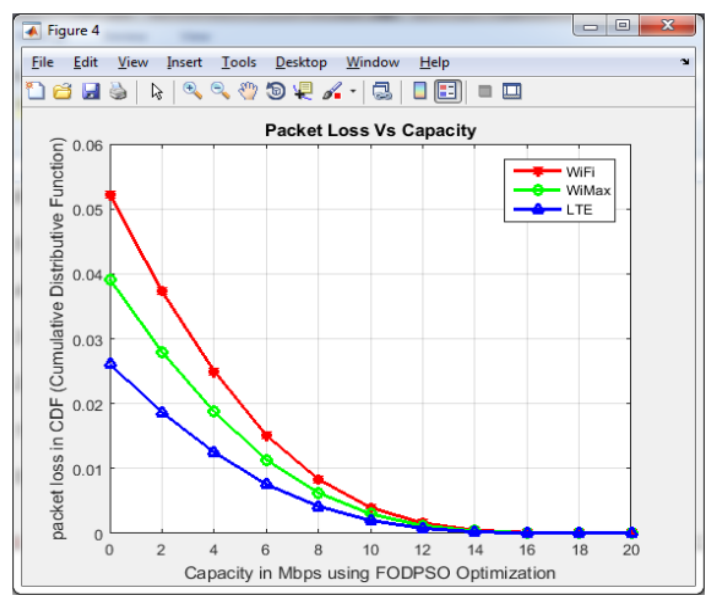

Fig. 10. Packet loss vs Capacity

\section{CONCLUSION}

The efficient routing of heterogeneous wireless network in terms energy and lifetime is important to design and evaluate the performance of the train. The Wi-Max, Wi-Fi and LTE networks are heterogeneously connected to improve the performance of the WSN network. The latency of the HSN is highly reduced to improve the speed of the data transmission in between network. The throughput of the HSN is highly improved by applying the FODPSO optimization algorithm. The Q-LEACH protocol is proposed to obtain the high range of routing to improve the performance of the HSN.

\section{REFERENCES}

[1] Jiyan Wu, Member, IEEE, Chau Yuen, "Bandwidth-Efficient Multipath Transport Protocol for Quality-Guaranteed Real-Time Video Over Heterogeneous Wireless Networks," IEEE TRANSACTIONS ON COMMUNICATIONS, VOL. 64, NO. 6, JUNE 2016.

[2] ZHENG Jie1, LI Jiandong2, "on Minimizing Delay with Probabilistic Splitting of Traffic Flow in Heterogeneous Wireless Networks," China Communications • December 2014

[3] Mohamed M. E. A. Mahmoud1, Xiaodong, "Secure and Reliable Routing Protocols for Heterogeneous Multihop Wireless Networks," 1045-9219/13/\$31.00 @ 2013 IEEE.

[4] Ali Al-Sherbaz, Sabah Jassim, "Private synchronization technique for heterogeneous wireless network (WiFi and WiMAX)," See discussions, stats, and author profiles for this publication at: https://www.researchgate.net/publication/238747321.

[5] Abhijit Sarma, Member, IEEE, Sandip Chakraborty, "Deciding Handover Points based on Context Aware Load Balancing in a WiFi-WiMAX Heterogeneous Network Environment," IEEE TRANSACTIONS ON VEHICULAR TECHNOLOGY.

[6] Yashuang Guo, Qinghai Yang, "Quality-Aware Streaming in Heterogeneous Wireless Networks," IEEE TRANSACTIONS ON WIRELESS COMMUNICATIONS, VOL. 16, NO. 12, DECEMBER 2017

[7] Michael J. Neely, "Energy Optimal Control for Time-Varying Wireless Networks," IEEE TRANSACTIONS ON INFORMATION THEORY, VOL. 52, NO. 7, JULY 2006.

[8] Mohammed Abo-Zahhad, Sabah M. Ahmed, "Mobile Sink based Adaptive Immune Energy Efficient Clustering Protocol for Improving the Lifetime and Stability Period of Wireless Sensor Networks," 1530-437X (c) 2015 IEEE.

[9] Zhao Han, Jie Wu, "A General Self-Organized Tree-Based Energy-Balance Routing Protocol for Wireless Sensor Network," IEEE TRANSACTIONS ON NUCLEAR SCIENCE, VOL. 61, NO. 2, APRIL 2014.

[10] Phuong Luong, Tri Minh Nguyen, "Energy-Efficient WiFi Offloading and Network Management in Heterogeneous Wireless Networks," 2169-3536 (c) 2016 IEEE.
[11] Rajarajan Sivaraj , Aravind Kota, "QoS-enabled Group Communication in Integrated VANET-LTE Heterogeneous Wireless Networks,"2011 IEEE $7^{\text {th }}$ international Conference on wireless and mobile computing.

[12] Eleni Stai1, Michail Loulakis2, "Congestion \& Power Control of Wireless Multihop Networks over Stochastic LTF Channels,"2015 IEEE Wireless communication and networking conference.

[13] Duc Chinh Hoang, "Real-Time Implementation of a Harmony Search Algorithm-Based Clustering Protocol for Energy-Efficient Wireless Sensor Networks," IEEE TRANSACTIONS ON INDUSTRIAL INFORMATICS, VOL. 10, NO. 1, FEBRUARY 2014.

[14] Yan Jiao and Inwhee Joe, "Energy-Efficient Resource Allocation for Heterogeneous Cognitive Radio Network based on Two-Tier Crossover Genetic Algorithm," JOURNAL OF COMMUNICATIONS AND NETWORKS, VOL. 18, NO. 1, FEBRUARY 2016

[15] Joohyung Lee, Nga T. Dinh, Ganguk Hwang, "Power-Efficient Load Distribution for Multihomed Services With Sleep Mode Over Heterogeneous Wireless Access Networks," IEEE TRANSACTIONS ON VEHICULAR TECHNOLOGY, VOL. 63, NO. 4, MAY 2014.

[16] Illsoo Sohn, Jong-Ho Lee, "Low-Energy Adaptive Clustering Hierarchy Using Affinity Propagation for Wireless Sensor Networks," 1089-7798 (c) 2015 IEEE.

[17] Amina Gharsallah, Nouri Omheni, "Network Selection in Heterogeneous Wireless System Environments," Nouri Omheni on 19 December 2017

[18] Yongjun Sun, Wenxin Dong and Yahuan Chen, “An Improved Routing Algorithm Based on Ant Colony Optimization in Wireless Sensor Networks," IEEE COMMUNICATIONS LETTERS, VOL. XX, NO. XX, XXX 2016

[19] Vlad Tabus, Dmitri Moltchanov, "Energy Efficient Wireless Sensor Networks Using Linear-Programming Optimization of the Communication Schedule," JOURNAL OF COMMUNICATIONS AND NETWORKS, VOL. 17, NO. 2, APRIL 2015

[20] Rejina Parvin,Vasanthanayaki C, "Particle Swarm Optimization based Clustering by Preventing Residual Nodes in Wireless Sensor Networks," $1530-437 X$ (c) 2015 IEEE. Personal use is permitted, but republication/redistribution requires IEEE permission.

\section{AUTHORS PROFILE}

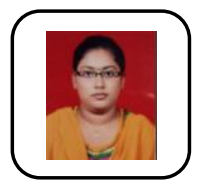

S.Priyanka is a research scholar and is pursuing her M.E degree in the Department of Communication Systems Engineering, Panimalar Engineering College, Anna University Chennai, India. She completed her B.E. in Electronics and Communication Engineering from Panimalar Institute of Technology, Anna University, Chennai, India. Her research areas include Wireless Communication, Robotics and Embedded technology. She has published paper on Smart Agriculture Robot and survey paper on Wireless Train Communication. The publication list includes national and international journals and conferences.

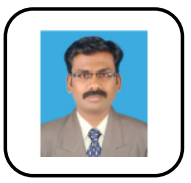

Leones Sherwin Vimalraj is a Professor in the Department of Electronics and Communication Engineering, Panimalar Engineering College, Chennai, India. He completed his B.E. in Electronics and Communication Engineering from Karunya Institute of Technology, Bharathiar University, India in 2001. He obtained his M.E. in Optical Communication Engineering (2004) from College of Engineering, Guindy, Anna University and PhD in Wireless Communication Engineering (2015) from Dr. MGR Educational and Research Institute, Deemed University, Chennai, India. He has around 15 years of experience as an academician, researcher and administrator. His research areas include Wireless Communication, Network engineering, computing and evolutionary algorithms. As the main and coauthor, he has more than 30 publications in his credit. The publication list includes national and international journals and conferences. 
Lydia $\mathbf{J}$ is a research scholar, and is pursuing her $\mathrm{PhD}$ degree in the Department of Electrical and Electronics, Anna University, Chennai, India. She has completed her B.E degree in Electrical and Electronics Engineering from Easwari Engineering College, Chennai, India. She obtained her M.E. in Power Electronics and Drives (2006) from Karunya Institute of Technology and SciencesDeemed University. She has around 13 years of experience in the education and research.Currently, she is an Associate Professor in the Department of Electrical and Electronics Engineering, Easwari Engineering College, Chennai, India and works in the fields of Power Electronics Electromagnetic Fields and Control Systems. She has published more than 20 research articles in International \& National Journals and Conferences. 